\title{
Surgical Reports of the Inferior Laryngeal Nerve and the Inferior Thyroid Artery in General Surgery and in ENT
}

\author{
Youssouf Sidibé ${ }^{1}$, Abdoulaye Kanté2,3*, Bréhima Bengaly², Siaka Diallo², Mariam Daou4, \\ Drissa Ouattara', Babou Ba ${ }^{3}$, Bréhima Coulibaly², Birama Togola², Drissa Traoré2, \\ Abdoul Wahab Haidara1, Boubacar Sanogo1, Nouhoum Ongoïba ${ }^{2,3}$
}

${ }^{1}$ ENT and Head and Neck Surgery Department CHU "Mother-Child”, The Luxembourg, Bamako, Mali

${ }^{2}$ Service of Surgery B. CHU Point G, Bamako, Mali

${ }^{3}$ Laboratory of Anatomy of the Faculty of Medicine and Odontostomatology, Bamako, Mali

${ }^{4}$ Service of Neurology, CHU Gabriel Touré, Bamako, Mali

Email: *kanteim@yahoo.fr

How to cite this paper: Sidibé, Y., Kanté, A., Bengaly, B., Diallo, S., Daou, M., Ouattara, D., Ba, B., Coulibaly, B., Togola, B., Traoré, D., Haidara, A.W., Sanogo, B. and Ongoiba, N. (2019) Surgical Reports of the Inferior Laryngeal Nerve and the Inferior Thyroid Artery in General Surgery and in ENT. Forensic Medicine and Anatomy Research, 7, 24-30.

https://doi.org/10.4236/fmar.2019.71005

Received: October 15, 2018

Accepted: December 20, 2018

Published: January 22, 2019

Copyright $\odot 2019$ by authors and Scientific Research Publishing Inc. This work is licensed under the Creative Commons Attribution International License (CC BY 4.0). http://creativecommons.org/licenses/by/4.0/

\begin{abstract}
Aim: The purpose of this study was to determine the frequency of the hurts of the inferior laryngeal nerve, according to its anatomical reports with the inferior thyroid artery during the thyroid surgery. Methodology: We realized a forward-looking and retrospective study from January, 1979 till December, 2017 in the service of surgery " $\mathrm{B}$ " to the University hospital of the Point G of Bamako and in the service of ENT and cervico-facial surgery of the Teaching Hospital "Mother-Child", the Luxembourg of Bamako (Mali). All the patients operated in both services for mild goiters were retained. Cancers and other thyroid pathologies were not included. The diagnosis of mild goiter was paused by the histological examination realized on all the surgical specimens. Results: On 2109 dissections of the inferior laryngeal nerve realized during the surgical operations on the thyroid, the frequency of lesion of the inferior laryngeal nerve was $1.09 \%$ ( 20 cases) when it passed dorsally with regard to the inferior thyroid artery (1837 cases) and when $4.04 \%$, it was transvascular or prevascular (272 cases). Conclusion: The prevascular route or transvascular of the inferior laryngeal nerve favors its lesion per operating.
\end{abstract}

\section{Keywords}

Anatomy, Inferior Laryngeal Nerve, Recurring Nerve, Thyroid Surgery, Goiter

\section{Introduction}

The surgery of the thyroid gland requires a perfect knowledge of the cervical 
anatomy: not only side and previous regions, but also pharyngo-laryngeal structures in their normal morphology and their possible anatomical variations. Particular importance in thyroid surgery is the arterial and venous vascularization of the gland, the peri-thyroid sheath, the situation of the parathyroid glands and the situation of the laryngeal nerves, superior and especially inferior [1].

The paralysis of the inferior laryngeal nerve is an iatrogenic grave complication which can lead important disorders of the phonation, the breath and the gulp. It can of this fact have important psychological echoes limiting then working capacities and social lives of the patients [2]. Thus, it is very important to know well the anatomy of the inferior laryngeal nerve of its origin about its ending, its situation and its reports in the thyroid region.

The route of the inferior laryngeal nerve is highly variable, and of this fact, there is no constant report with the close anatomical structures that it crosses [2]. That is why there are numerous surgical techniques of location of the inferior laryngeal nerve during the thyroid surgery. The inferior laryngeal nerve is not as vulnerable as it does not admit in its contact a careful dissection. The "danger to perceive" it for a long time put forward to justify its not-dissection is not accepted anymore by most of the surgeons [3].

It is the variability of the situation of the nerve, rather than its vulnerability, that is responsible for the frequency with which it can be hurt [4]. So the iatrogenic wound of the inferior laryngeal nerve or its branches will be avoided at best for, with caution, a search, identification and an exhibition of the nerve during its route [4].

The purpose of this work is to study the report between the anatomical variations of the inferior laryngeal nerve and the operating hurts of this nerve, to determine the frequency of the hurts of the inferior laryngeal nerve according to the number of its branches in the thyroid changing room and according to its position with regard to the inferior thyroid artery.

\section{Materials and Methods}

We realized a retrospective study from January, 1979 till December 2011 and prospective from January, 2012 till December, 2017 (that is 38 years of study) in the service of surgery " $\mathrm{B}$ " to the University hospital of the Point G of Bamako and in the service of ENT and cervico-facial surgery of the CHU Mother-child "the Luxembourg" of Bamako (Mali).

All the patients operated in both services for mild goiters were retained. Were not included in this study: the cancers of the thyroid, the thyroiditis, the reoperations on thyroid, isthmectomies, strumitis and the sick who had a lesion of the inferior laryngeal nerve in preoperative.

The diagnosis of mild goiter was paused by the histological examination realized on all the surgical specimens.

The group of hyperthyroid patients was handled medically with antithyroid ones of synthesis to have aeuthyroidy before being scheduled for the surgical operation. 
A complete clinical examination, a dosage of free T4, ultrasensitive TSH, a preoperative biological balance sheet and a cervical ultrasound were made at all the patients. The patients all had a systematic laryngoscopy meadow and operating comment between the 3rd and the 7th day to study the mobility of vocal cords. The sick were clinically followed in post-operative between the day of the intervention and the 3rd post-operative day, and between the 30th and the 60th post-operative day.

In case of disorder of the mobility of a vocal cord, the laryngoscopy was repeated between the 30 th and the 60 th post-operative day.

For the operation, the sick person is installed in dorsal decubitus; the head is in extension rest on a masthead in gel of silicone. A block placed under the shoulders facilitates this extension. The latter are lowered at the most to clear well the neck. The way at first was a previous transversal cervicotomy type Kocher.

After section of the muscle cutaneous of the neck, the previous jugular veins are ligatured and cut. A detachment is made between the venous plan ventrally, the muscles sternocleidomastoid dorsally and laterally, and the sternothyroid muscles dorsally and medially. The detachment reaches cranially the inferior edge of the thyroid cartilage and caudally the upper edge of the manubrium sternal. A median vertical section is then realized up to the thyroid capsule.

No muscle is cut in this way at first. The thyroid capsule is opened. The inferior laryngeal nerve is spotted in a triangle lined by the primitive carotid outside, the trachea inside and the thyroid at the top. It is white sinuous, traveled by a very visible arterial. The dissection of the deep face of the lobe is led from the outside inside, until the penetration of the nerve in the larynx, to the inferior edge of the cricoid cartilage. To the left, the nerve walks in the angle tracheo-œsophageal. To the right it is in more side position. Its situation with regard to the inferior thyroid artery is unpredictable: crossing above, below or between branches.

The branches of the inferior laryngeal nerve were considered multiple when two branches or more detected in the thyroid changing room made to the muscle inferior constrictor of the larynx.

We defined by lesion of the inferior laryngeal nerve, any immobility or paresis of a vocal cord.

The studied parameters were the following ones: the sociodemographic data, the anatomopathologic data, the global frequency of lesion of the inferior laryngeal nerve, the frequency of the lesion of the inferior laryngeal nerve according to the number of branches of division of the inferior laryngeal nerve in the thyroid changing room, the frequency of the lesion of the inferior laryngeal nerve according to its report with the inferior thyroid artery.

The data were seized and analyzed on the software Ear information (version 6). The test of $\mathrm{X}^{2}$ and Student were used to compare our results with those of the literature. The threshold of meaning was fixed to 0.05 . 


\section{Results}

During the study period, we dissected 2109 inferior laryngeal nerves during thyroid surgery.

\subsection{Sociodemographic Data}

The average age of the patients was of 37 years with extremes of 10 years and 78 years, the age bracket $40-49$ years represented 33.6\%. It was 136 men and 919 women (Figure 1 and Figure 2), 282 (26.7\%) lived in Bamako whereas 773 (73.3\%) came from 11 regions of Mali and 756 (71.7\%) had a low socioeconomic level.

\subsection{Anatomo-Pathological Data}

The histological examination was made at all the sick. We found a goiter colloid in $77 \%$. The reserved etiologies are represented in Table 1.

\subsection{Global Frequency of Lesion of the Inferior Laryngeal Nerve}

We looked for to identify the inferior laryngeal nerve in 2184 times; it was seen and dissected in 2109 cases (96.5\%). It was not seen 75 cases (3.4\%).

The global frequency of lesion of the inferior laryngeal nerve was 33 cases $(1.6 \%)$ on 2109 seen inferior laryngeal nerves.

\subsection{Frequency of the Lesion of the Inferior Laryngeal Nerve according to the Number of Branches of Division of the Inferior Laryngeal Nerve in the Thyroid Changing Room (Table 2)}

The accidental lesion of the inferior laryngeal nerve arose in 27 cases (1.34\%) when there were troncular (2006 cases). On the other hand it arose in 2 cases (1.94\%) when the nerve was multiple (103 cases). The difference was not statistically significant $(p=0.08)$. Thus the lesion of the inferior laryngeal nerve it peroperatoire is not influenced by the number of branches of division of the inferior laryngeal nerve.

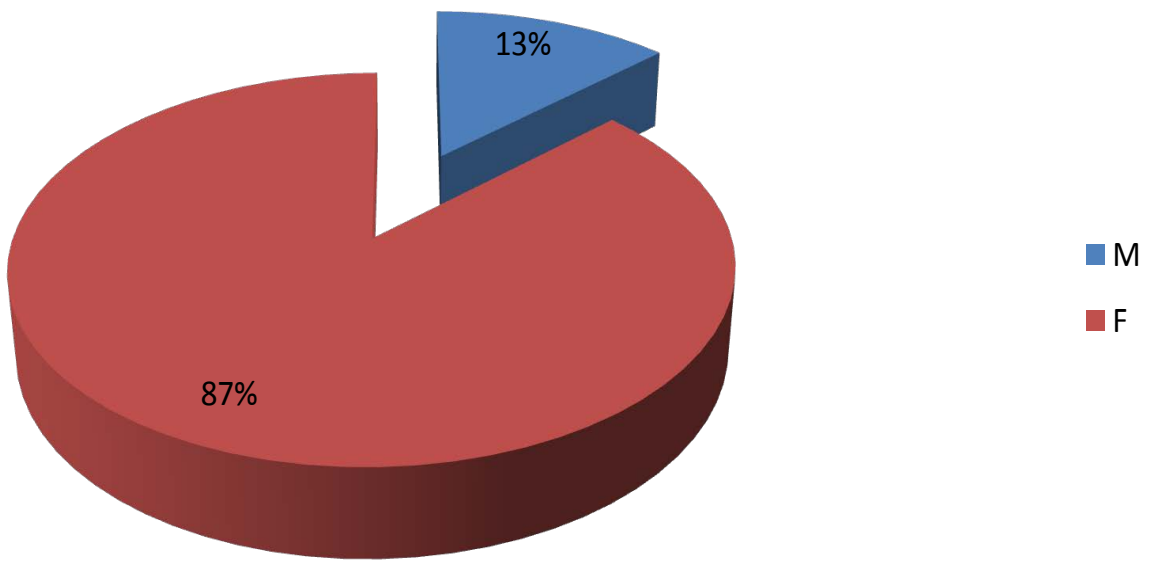

Figure 1. Distribution of the patients according to the sex. 


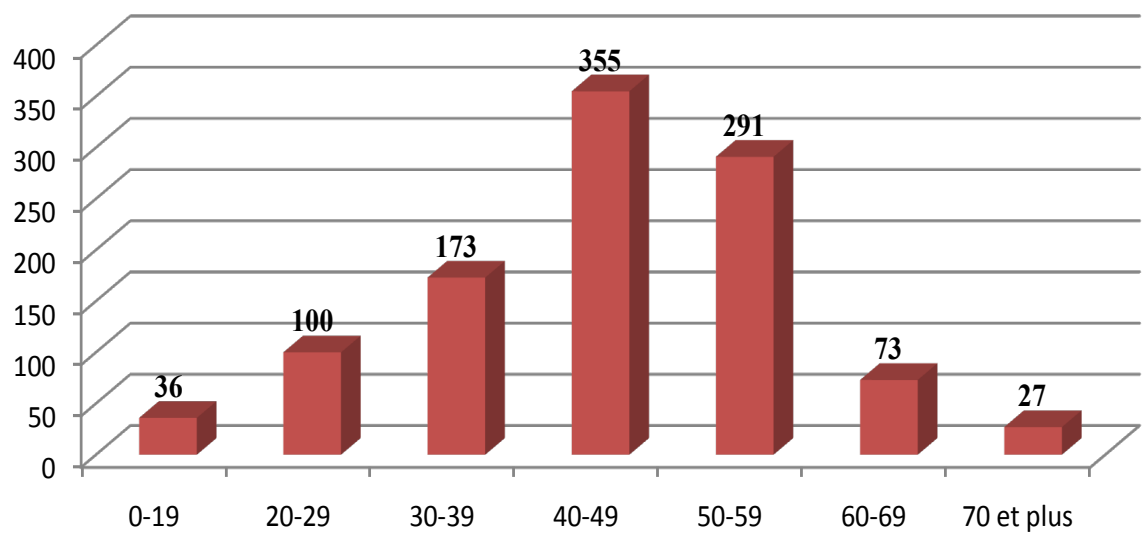

Figure 2. Distribution of the patients according to the age bracket.

Table 1. Etiologies of goiters.

\begin{tabular}{ccc}
\hline Etiologies of the goiters & Effectif & Percentage \\
\hline Multi-goiter - heterosexual - nodulaire toxin & 272 & $25.8 \%$ \\
Sickperson of Basedow & 151 & $14.3 \%$ \\
Basedow if goiter & 61 & $5.8 \%$ \\
Hyperthyroid unique nodule & 35 & $3.3 \%$ \\
Hypofunctional goiter & 536 & $50.8 \%$ \\
Total & 1055 & $100 \%$ \\
\hline
\end{tabular}

Table 2. Frequency of the hurts according to the number of branches of division of the inferior laryngeal nerve (ILN) in the thyroid changing room.

\begin{tabular}{cccccc}
\hline \multirow{2}{*}{\begin{tabular}{c} 
Lesion of the ILN Number of $\begin{array}{c}\text { Not } \\
\text { branches of the ILN }\end{array}$ \\
\cline { 2 - 5 }
\end{tabular}} & \multicolumn{3}{c}{ Yet } & \multirow{2}{*}{ Total } \\
Unique & 1979 & 98.66 & 27 & 1.34 & 2006 \\
Multiple & 101 & 98.06 & 2 & 1.94 & 103 \\
Total & 2080 & 98.62 & 29 & 1.38 & 2109 \\
\hline
\end{tabular}

\subsection{Frequency of the Lesion of the Inferior Laryngeal Nerve according to its Report with the Inferior Thyroid Artery (Table 3)}

In the cases where the inferior laryngeal nerve passes dorsally with regard to the inferior thyroid artery (1837 cases), it was hurt in 20 times or (1.09\%). But in the cases where the inferior laryngeal nerve crosses it transvascular (between the branches of the artery) or in prevascular (272 cases), its lesion arose in 11 times (4.04\%). The difference is significant $(\mathrm{p}=0.0002)$. Thus the report between the inferior laryngeal nerve and the inferior thyroid artery influences the arisen of an operating lesion of this nerve. The prevascular route or transvascular of the inferior laryngeal nerve favors its lesion per operating. 
Table 3. Frequency of the hurts of the inferior laryngeal nerve (ILN) according to its report with the inferior thyroid artery (InfThyr. Art.).

\begin{tabular}{cccccc}
\hline \multirow{2}{*}{\begin{tabular}{c} 
Lesion of ILN report of the ILN with $\begin{array}{c}\text { Not } \\
\text { the InfThyr. Art }\end{array}$ \\
\cline { 2 - 5 } Retro-vascular
\end{tabular}} & \multicolumn{3}{c}{ Yes } & Total \\
\hline number & $\%$ & number & $\%$ & \\
Meadow and transvascular & 1817 & 98.91 & 20 & 1.09 & 1837 \\
Total & 261 & 95.96 & 11 & 4.04 & 272 \\
\hline
\end{tabular}

\section{Discussion}

On 2109 seen and dissected inferior laryngeal nerves, we found 33 hurts (1.6\%). This frequency is identical to that reported by Koumare [5], $(\mathrm{p}=0.8)$; but it is statistically inferior than those reported by Blondeau [6], and of Soustelle [7] (p $=0.0002)$. This difference could give some explanation by the fact that we eliminated many factors favoring the lesion of the inferior laryngeal nerve (cancer, thyroiditis, reoperations on thyroid).

On 2109 inferior laryngeal nerves (right or left) seen and dissected, we found a premature fork of the nerve in $4.8 \%$ of the cases. This frequency is comparable to that reported by Koumaré.

On 2109 seen and dissected inferior laryngeal nerves, we found a position retrovascular of the inferior laryngeal nerve in $98.91 \%$ of the cases. Koumaré, on 1133 cases found that the inferior laryngeal nerve was retrovascular in $98.89 \%$. Blondeau and Berlin, on 366 cases and 140 cases respectively brought back a position retrovascular of the inferior laryngeal nerve in $54.1 \%$ and $52.1 \%$. Our frequency of position retrovascular of the inferior laryngeal nerve is identical to that brought back by Koumaré but statistically different to those brought back by Blondeau and Berlin [8] ( $p=0.001 ; p=0.0002)$. We did not find explanation to it.

Very few studies were realized on the report enter the lesion per operating of the inferior laryngeal nerve and the premature division of this nerve. But several authors agree to say that the prevascular position of the inferior laryngeal nerve favors its lesion per operating [9].

\section{Conclusions}

Recurrential complications are rare in thyroid surgery if the inferior laryngeal nerve is dissected (1.38 in our study).

The operating hurts are not increased by the number of branches of division of the inferior laryngeal nerve. On the other hand, its lesion is influenced by its route meadow or retrovascular.

\section{Authorization of the Ethics Committee}

We, undersigned, authors of this article, give evidence to have received the authorization of the Ethics Committee of the Faculty of Medicine of Bamako. 


\section{Conflicts of Interest}

We, authors of this article, declare that there is no conflict of interests.

\section{References}

[1] Lubrano, D., Levy-Chazal, N., Araya, Y. and Avisse, C. (2002) Research for the Lower or More Recurring Laryngeal Nerve during a Thyroid Lobectomy. Annals of Surgery, 127, 68-72.

[2] Rosta, L., Avenia, N. and Bernante, P. (2004) Complications of Thyroid Surgery; Analysis of a Multicentric Study on 14934 Patients Operated in Italy over 5 Years. Word Journal of Surgery, 28, 271-276.

[3] Kang, A., Grant, C. and Thompson, G. (2002) Current Treatment of Nodular Goiter with Hyperthyroidism (Plummer's Disease): Surgery versus Radioiodine. Surgery, 132, 916-923. https://doi.org/10.1067/msy.2002.128691

[4] Sanogo, Z.Z., Koïta, A.K., Koumaré, S., Keïta, S., Saye, Z., Camara, M., et al. (2012) Surgical Care of the Hyperthyroid Goiters in Bamako. Journal of Medicine in Mali, 27, 1-4.

[5] Koumaré, A.K., Ongoïba, N., Sissoko, F., Berété, S., Traore, A.K., Sidibé, Y., et al. (2002) Inferior Laryngeal Nerve: Anatomy and Operating Hurts. E-Memoire of the Academy of Surgery, 1, 8-11.

[6] Blondeau, P. (1971) Surgical Reports of the Recurring Nerve and the Thyroid Artery. Journal of Surgery, 102, 397-414.

[7] Soustelle, J. and Vincent, F. (1970) The Recurrentiel Risk in Thyroid Surgery. Simep Publishing (Editions), Lyon.

[8] Berlin, D.D. (1935) The Recurrent Laryngeal Nerves in Total Ablation of the Normal Thyroid Gland. Journal of Gynecology and Obstetrics, 60, 19-26.

[9] Soulet, J. (2000) The Recurrentiel Complications of the Surgery of the Thyroid Body. Thesis Medicine Poitiers, No. 97. 